\title{
Overcoming the Influence of Human Shadowing and Obstacles via Modified Building Using Frequency Selective Wallpapers for $60 \mathrm{GHz}$
}

\author{
Nidal Qasem, Emran Ali Aldorgam, and Hadeel Yaseen Alzou'bi \\ Department of Electronics and Communications Engineering, Al-Ahliyya Amman University, 19328, Amman, Jordan
}

\begin{abstract}
The growing demand for a very high speed and bandwidth over wireless communications makes the millimeter wave (mm-wave) "60 GHz" a great choice to be studied and used in order to achieve this desire. While the propagating signal of this frequency can be easily blocked by any obstacle or body that faces it. This paper aims to solve this problem by using the SL (square loop) FSS (frequency selective surfaces) in such a way to provide a stronger reflected signal to those shadowed positions as a result from the variety of the signal paths and enhancing the received signal power. So, minimizing the effect of human bodies that can attenuate $60 \mathrm{GHz}$ signal.
\end{abstract}

Keywords: $60 \mathrm{GHz}$, band-stop, frequency selective surface, human modeling, millimeter wave.

\section{Introduction}

The development of wireless communications is stimulated by the consumer desire for access to information and entertainment. While contemporaneity unlicensed systems support moderate levels of wireless data traffic, as seen in Bluetooth and WLANs (wireless local area networks). Current technology is unable to supply data rates comparable to wired standards like GbE (Gigabit Ethernet) and HDMI (high definition multimedia interface) [1]. As result of that data rates or bandwidths are never enough, while the wireless multimedia distribution market is ever growing [2]. So, an obvious solution to this problem is to resort to the $60 \mathrm{GHz}$ band, where bandwidth is abundantly available [3].

Since the wavelength at $60 \mathrm{GHz}$ is $5 \mathrm{~mm}$ which is too small, obstacles such humans and furniture can block links easily. For example, blockage by a human can affect the link budget communication by 20-30 $\mathrm{dB}$. So, movements of humans might cause a

Corresponding author: Nidal Eyad Qasem, Ph.D., assistant professor, research fields: antennas, frequency selective surfaces, propagation control within buildings, ultra-wide band. discontinuous blockage of millimeter wave links [4]. So, this paper suggests a novel technique that will control the propagation of indoor wireless environment at $60 \mathrm{GHz}$ band by blocking the $60 \mathrm{GHz}$ signal from propagating inside the area of interest using SL FSS to provide a stronger reflected signal as well as providing multipath propagation due to the variety of signal paths.

This paper shows an introduction of FSS. ECM (equivalent circuit model) analysis technique is mentioned in details and applied on the SL FSS. Next, a scenario with only one room is presented with and without SL FSS to check its effect on improving the received signal power in presence of humans. Then, another scenario is created inside corridor and performed without SL FSS, with SL FSS and PEC (perfect electric conductor), and with $45^{\circ}$ inclined PEC. Finally, the open area scenario is studied in the presence and absence of SL FSS to show its effect on the mean time of arrival and delay spread values.

\section{Frequency Selective Surfaces}

FSSs (frequency selective surfaces) are planar 
periodic structures which exhibit reflection and/or transmission properties as a function of frequency. The surface consists of thin conducting elements, usually printed on dielectric substrates for support. In general, the FSS structures can be divided into patch-type or aperture-type metallic elements, being periodic in either a one or two dimensional array. Based on the element geometry, FSSs have four operation modes: low- pass, high-pass, band-pass, and band-stop filter [5]. FSS is now used in many fields in military applications, antennas, medical applications, and wireless communications security [6-12].

FSSs have many shapes and geometries. The most common shapes are: square loops, dipoles, tripoles, cross dipoles, Jerusalem crosses, and rings [5].

Previous researches showed that the SL FSS has the best performance and stability to work within the angular sensitivity, cross polarization TE (transverse electric) or TM (transverse magnetic) modes, and small band separation [13].

In order to test SL FSS, ECM has been considered as the simple method. The first usage of ECM technique and first applied to frequency selective circuits was by Anderson [14]. The advantage of ECM is that it can quickly characterize the FSS response with varying element dimensions. On other hand, ECM cannot be used to predict the performance of the sophisticated shapes other than simple shapes like SL FSS [15].

The frequency response in SL FSS is determined by the element dimensions ( $\mathrm{p}, \mathrm{d}, \mathrm{s}, \mathrm{g}$ ), as shown in the Fig. 1. Where:

$\mathrm{p}$ : is the separation period;

$\mathrm{d}$ :is the dimension of the loop;

$\mathrm{s}$ : is the width of the conducting strip;

$\mathrm{g}$ : is IES (inter-element spacing).

These parameters determine the location of the $f_{r}$ (resonant frequency). The parameter $\mathrm{g}$ controls the FSS angular performance [13]. Previous studies have suggested that a bigger square loop in size generally effective at a lower $f_{r}$ and a smaller g ensures stable $f_{r}$ with varying incidence angles [16].

ECM technique imposes that the interaction between incident wave and the FSS is represented as a wave propagating through a transmission line, with shunt lumped circuit impedances. The shunt impedance could be inductive or capacitive relying on whether the polarization of the incident signal is parallel or perpendicular to the strip. As seen from Fig. 2, the two adjacent strips are approximated as one strip with width (w) equal to (2s) [17].

The normalized shunt inductive reactance expression of the inductive strip was given as Ref. [18]:

$$
\begin{gathered}
X_{T E}=\frac{\omega_{o} L}{Z_{o}}= \\
\frac{p \cos (\theta)}{\lambda}\left[\ln \left(\csc \left(\frac{\pi w}{2 p}\right)\right)+G(p, w, \lambda, \theta)\right](1 . \mathrm{a}) \\
X_{T M}=\frac{p \sec \emptyset}{\lambda}\left[\ln \left(\csc \left(\frac{\pi w}{2 p}\right)\right)+G(p, w, \lambda, \emptyset)\right](1 . \mathrm{b})
\end{gathered}
$$

The normalized shunt capacitive susceptance expression was given as Ref. [15] by Lee:

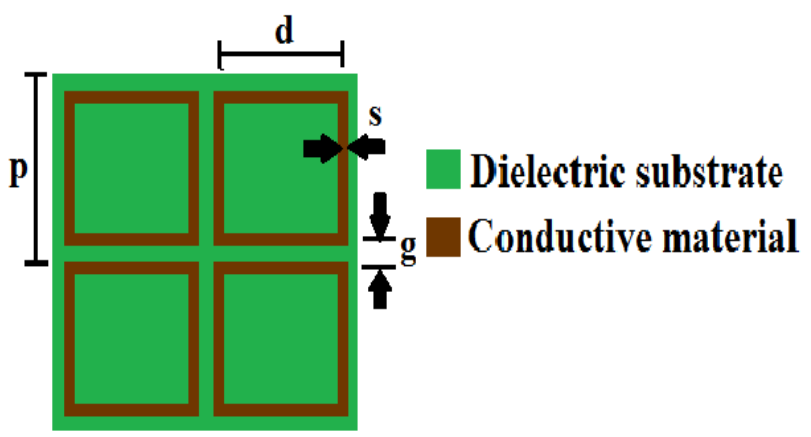

Fig. 1 Array of SL FSS elements.

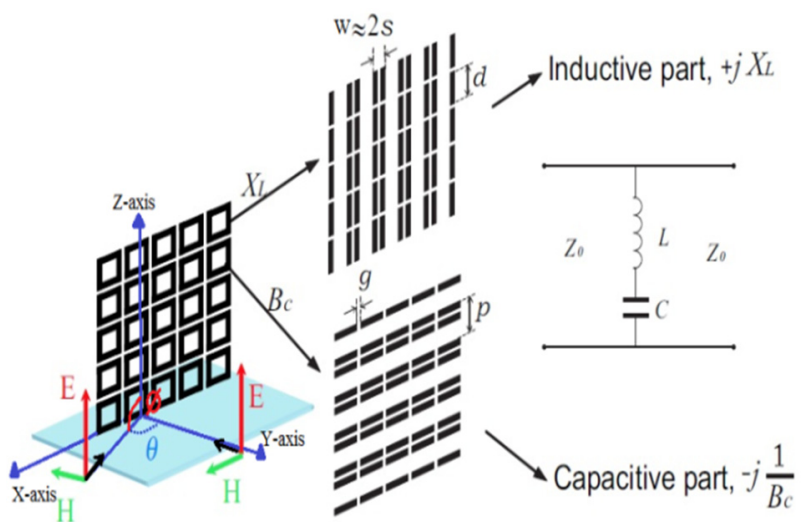

Fig. 2 Inductive and capacitive part of the SL FSS shape [11]. 


$$
\begin{gathered}
B_{T E}=\frac{\omega_{o} C}{Y_{o}}=\frac{4 p \sec (\theta)}{\lambda}\left[\ln \left(\csc \left(\frac{\pi g}{2 p}\right)\right)+\right. \\
G(p, g, \lambda, \theta)] \varepsilon_{e f f} \\
B_{T M}=4 F(p, g, \lambda)= \\
\frac{4 p \cos \phi}{\lambda}\left[\ln \left(\csc \left(\frac{\pi g}{2 p}\right)\right)+G(p, g, \lambda, \emptyset)\right] \varepsilon_{e f f}(2 . \mathrm{b})
\end{gathered}
$$

where $\mathrm{G}$ is the correction term:

$$
\begin{gathered}
G\left(p, w, \lambda, \begin{array}{l}
\theta \\
\emptyset
\end{array}\right)= \\
\frac{0.5\left(1-\beta^{2}\right)^{2}\left[\left(1-\frac{\beta^{2}}{4}\right)\left(A_{1+}+A_{1-}\right)\right]+4 \beta^{2} A_{1+} A_{1-}}{\left(1-\frac{\beta^{2}}{4}\right)+\beta^{2}\left(1+\frac{\beta^{2}}{2}-\frac{\beta^{4}}{8}\right)\left(A_{1+}+A_{1-}\right)+2 \beta^{6} A_{1+} A_{1-}} \\
G(p, g, \lambda, \theta)= \\
\frac{0.5\left(1-\beta^{2}\right)^{2}\left[\left(1-\frac{\beta^{2}}{4}\right)\left(A_{1+}+A_{1-}\right)\right]+4 \beta^{2} A_{1+} A_{1-}}{\left(1-\frac{\beta^{2}}{4}\right)+\beta^{2}\left(1+\frac{\beta^{2}}{2}-\frac{\beta^{4}}{8}\right)\left(A_{1+}+A_{1-}\right)+2 \beta^{6} A_{1+} A_{1-}}
\end{gathered}
$$

where

$$
\begin{gathered}
A_{1 \pm}^{T E}=\frac{1}{\sqrt{\left(\frac{p \sin \theta}{\lambda} \pm 1\right)^{2}-\frac{p^{2}}{\lambda^{2}}}}-1 \\
A_{1 \pm}^{T M}=\frac{1}{\sqrt{1-\frac{p^{2} \cos \phi}{\lambda^{2}}}}-1
\end{gathered}
$$

and

$$
\beta=\sin (0.5 \pi w / p)
$$

or

$$
\beta=\sin \left(\frac{0.5 \pi g}{p}\right)
$$

where $Z_{o}=377 \Omega, \theta$ is the incidence angle, $\lambda$ is the wavelength in air at operating frequency, $w$ is equal to $2 \mathrm{~s}$ in this calculation, and $\varepsilon_{e f f}$ is the effective permittivity of the surrounding dielectric substrate that influences the capacitance value.

Equations, which are presented in this section, have shown that the FSS response is a function of square loop element dimensions, incidence angles, and the dielectric material which holds and gives support for the FSS.

In addition, these equations are valid for $[p(1+$ $\sin \theta)<\lambda]$ for TE wave incidence and $[p(\cos \theta)<$ $\lambda]$ for $\mathrm{TM}$ wave incidence for a given range of incidence angle.

The equivalent impedance of the SL FSS is given by:

$$
Z_{F s s}=j\left(X_{L}-\frac{1}{B_{c}}\right)
$$

Hence, the normalized impedance $\left(Z_{n}\right)$ for the transmission line circuit, as illustrated in Fig. 3, can be determined by:

$$
Z_{n}=\frac{Z_{F s s}}{Z_{o}}=j\left(\frac{X_{L}}{Z_{o}}-\frac{1}{B_{c} Z_{o}}\right)=j\left(\frac{X_{L}}{Z_{o}}-\frac{Y_{o}}{B_{c}}\right)
$$

By using the $A B C D$ matrix, the transmission and reflection coefficients for the SL FSS can be determined. As it is known that an ABCD matrix can represent any given network; based on the $\mathrm{ABCD}$ parameters, the transmission coefficient $\left(\mathrm{S}_{21}\right)$ and the reflection coefficient $\left(S_{11}\right)$ can be determined [11]. For example, for a T-network as illustrated in Fig. 3, the $\mathrm{ABCD}$ matrix is presented by:

$$
\left[\begin{array}{ll}
A & B \\
C & D
\end{array}\right]=\left[\begin{array}{cc}
1+\frac{Z_{1}}{Z_{3}} & Z_{1}+Z_{2}+\frac{Z_{1} Z_{2}}{Z_{3}} \\
\frac{1}{z_{3}} & 1+\frac{Z_{2}}{Z_{3}}
\end{array}\right]
$$

The scattering S-matrix is defined as:

$$
\left[\begin{array}{ll}
S_{11} & S_{12} \\
S_{21} & S_{22}
\end{array}\right]=\left[\begin{array}{cc}
\frac{A+B-C-D}{\Delta} & \frac{2(A D-B C)}{\Delta} \\
\frac{2}{\Delta} & \frac{-A+B-C+D}{\Delta}
\end{array}\right]
$$

where: $\Delta=\mathrm{A}+\mathrm{B}+\mathrm{C}+\mathrm{D}$.

For the SL FSS, $Z_{1}=Z_{2}=0$ and $Z_{3}=Z_{n}$ as defined in Eq. (9). Therefore, based on Eq. (10) and Eq. (11), the transmission coefficient $\mathrm{S}_{21}$ and reflection coefficient $S_{11}$ for the FSS can be evaluated.

Because of symmetry $S_{11}=S_{22}$ and $S_{12}=S_{21}$. The $A B C D$ matrix can be expressed as a function of $S_{21}$ and $\mathrm{S}_{11}$ as:

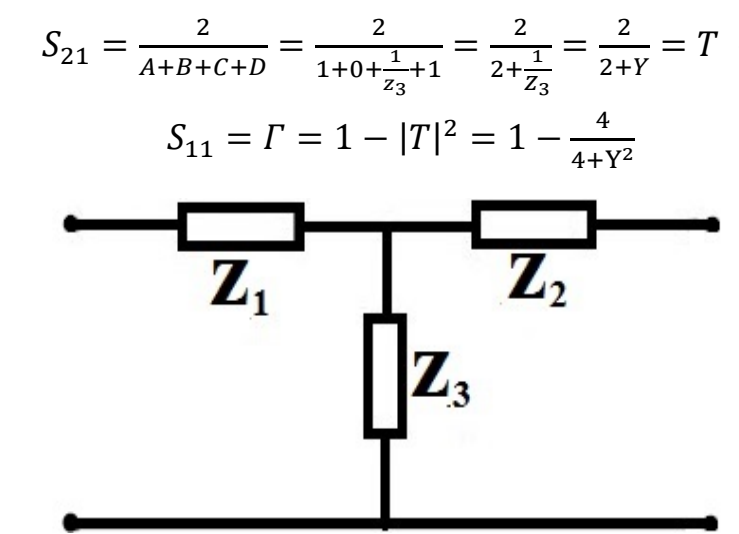

Fig. 3 Transmission line which can be represented by ABCD matrix. 


\section{Simulation Results Using MATLAB}

MATLAB is a system used to solve the theoretical equations for ECM. For SL elements, resonance occurs when each half loop acts as a dipole [19, 20]. The rule of thumb in designing the loop circumference is to make it approximately equal the resonant frequency wavelength $(60 \mathrm{GHz})$. Basic design rules for SL FSS element dimensions are given along with equations for the ECM. Where its dimensions are: $\mathrm{p}=1.4 \mathrm{~mm}, \mathrm{~s}=0.1 \mathrm{~mm}, \mathrm{~d}=1.2 \mathrm{~mm}, \mathrm{~g}=$ $0.2 \mathrm{~mm}$, and $\varepsilon_{\text {eff }}=\frac{\varepsilon_{r}+1}{2}=2$.

Figs. 4 and 5 show the frequency response for TE-mode and TM-mode incidence respectively. The result at normal incident between them is identical because the SL FSS shape has a symmetric geometry.

\section{Simulation Results Using CST (Computer Simulation Technology) MWS (Microwave Studio)}

As discussed in Section 3, the ECM has been used just as a design tool which helped to quickly identify the SL FSS frequency response. As shown in Fig. 6, SL FSS is tested again by using CST-MWS for a range of different incidence angles from $0^{\circ}$ to $60^{\circ}$.

The $\mathrm{S}_{21}$ is presented for TE and TM modes, as shown in Figs. 7 and 8 respectively. The $S_{11}$ is also presented for TE and TM modes, as shown in Figs. 9 and 10 respectively.

These figures are expressed in a range of frequencies from 50 to $70 \mathrm{GHz}$ and a range of incident angles from $0^{\circ}$ to $60^{\circ}$. The copper thickness is 0.07 $\mathrm{mm}$. The dielectric material is Arlon AD 300 which has $0.1 \mathrm{~mm}$ thickness and its relative permittivity is 3 .

In order to design a suitable FSS, the attenuation value at the resonant frequency, $60 \mathrm{GHz}$, must exceed $-25 \mathrm{~dB}$ to kill the signal [9].

As shown in Figs. 7 and 8, the transmission coefficients for both TE and TM modes are more than this range. So, the signal will be dead when it tries to penetrate/go out of the area of interest.
The reflection coefficients shown in Figs. 9 and 10 are even much smaller than $-0.05 \mathrm{~dB}$ at $60 \mathrm{GHz}$; this will let SL FSS acts as a perfect reflector inside the area of interest as stated before.

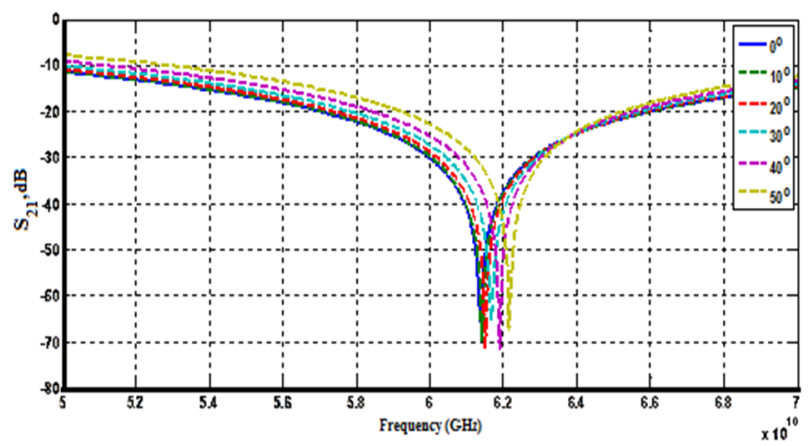

Fig. 4 The theoretical frequency response for TE-mode incidence results based on the ECM equations.

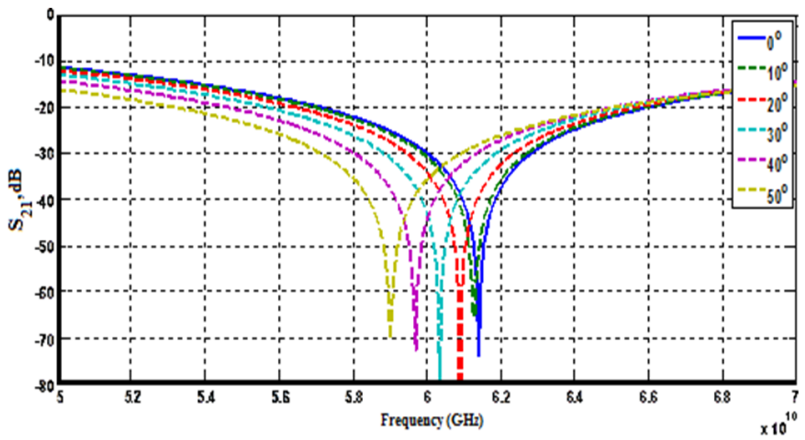

Fig. 5 The theoretical frequency response for TM-mode incidence results based on the ECM equations.

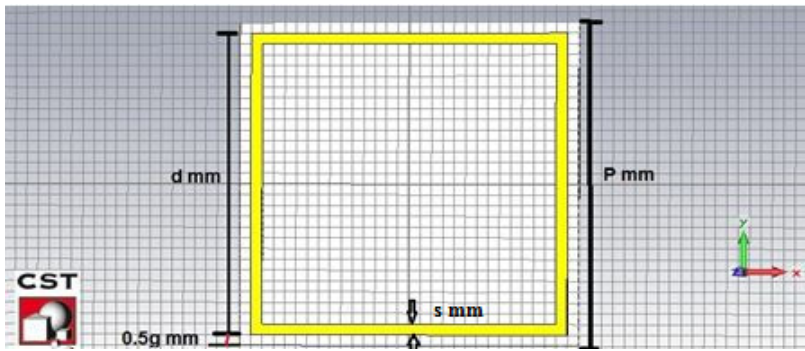

Fig. 6 The SL FSS dimensions, where $p=1.4 \mathrm{~mm}, \mathrm{~g}=0.2$ $\mathrm{mm}, \mathrm{d}=1.2 \mathrm{~mm}$, and $\mathrm{s}=0.1 \mathrm{~mm}$.

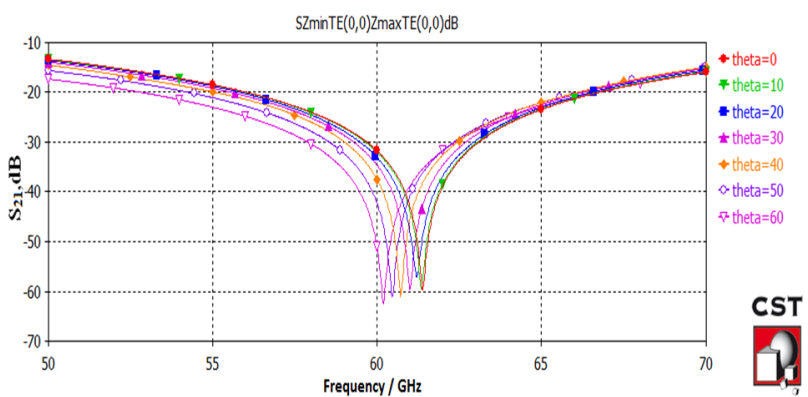

Fig. 7 Transmission response $\left(S_{21}\right)$ for TE-mode of $\mathrm{SL}$ FSS. 
Using Frequency Selective Wallpapers for $60 \mathrm{GHz}$

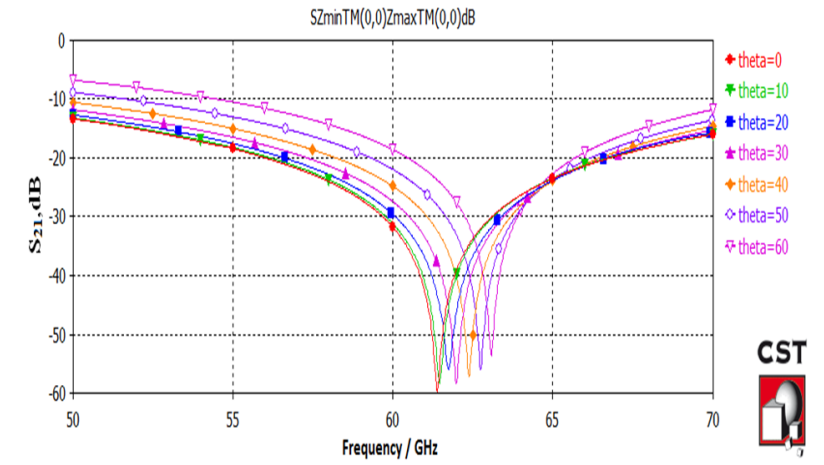

Fig. 8 Transmission response $\left(S_{21}\right)$ for TM-mode of SL FSS.

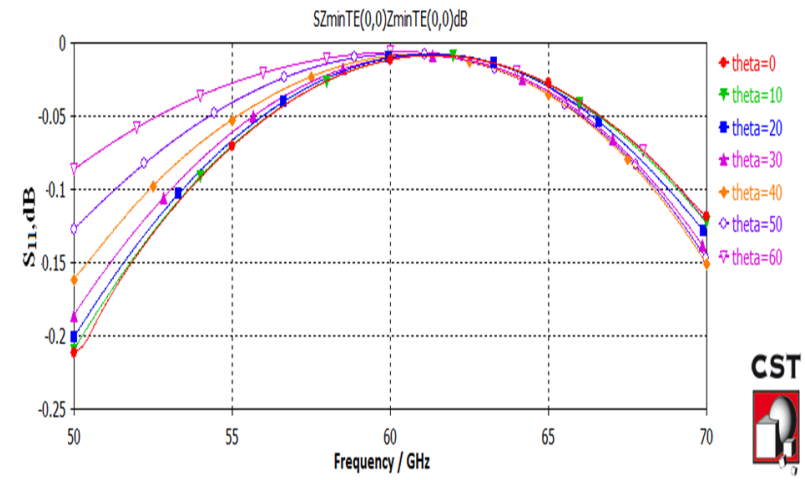

Fig. 9 Reflection response $\left(S_{11}\right)$ for TE-mode of SL FSS.

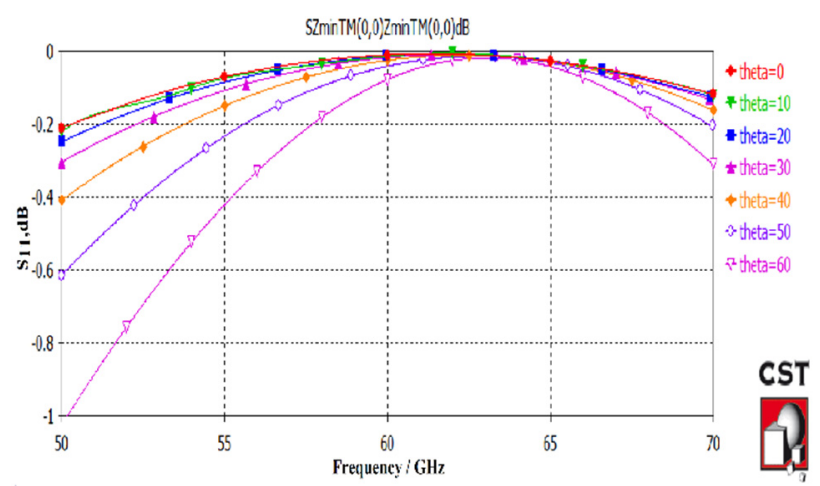

Fig. 10 Reflection response $\left(S_{11}\right)$ for TM-mode of SL FSS.

Fig. 11 shows how the designed SL FSS works as band-stop filter for the $60 \mathrm{GHz}$. It blocks the waves of $60 \mathrm{GHz}$ from port 1 to port 2. So, signal will be attenuated at port 2 .

Fig. 12 shows how the designed SL FSS works for a frequency rather than the frequency of interest; it will pass the waves normally from port 1 to port 2 .

\section{Human Modeling at $60 \mathrm{GHz}$}

The mm-wave range requires careful characterization of the antenna/human body interaction in order to evaluate, and possibly minimize, the variations of antenna characteristics and exposure levels induced to the body. To this end, knowledge of the dielectric properties of the human tissues is crucial for the numerical modeling and development of experimental phantoms. Any variations of the dielectric properties could directly impact the theoretical and experimental results [21]. Table 1 lists some typical values of the complex permittivity, at $60 \mathrm{GHz}$, for human skin, fat, muscle, and pure water at $20^{\circ} \mathrm{C}$ [22]. Where:

$$
\varepsilon_{c}=\varepsilon_{r}-j \frac{\sigma}{\omega \varepsilon_{o}}
$$

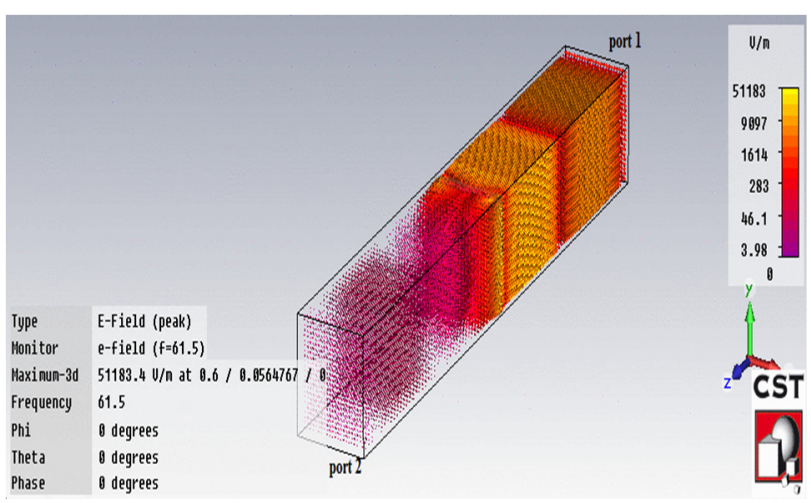

Fig. 11 Signal propagation animation, from port 1 to port 2 , at $60 \mathrm{GHz}$.

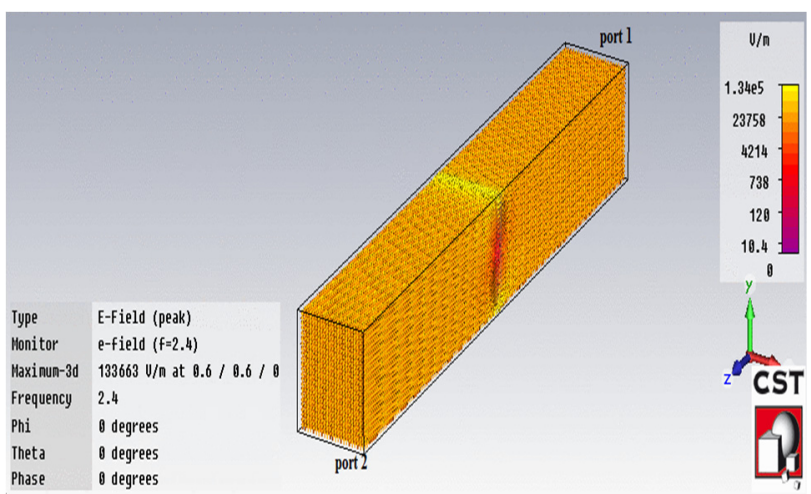

Fig. 12 Signal propagation animation, from port 1 to port 2, at $2.4 \mathrm{GHz}$.

Table 1 Complex permittivity at $60 \mathrm{GHz}$.

\begin{tabular}{ll}
\hline Human tissues & $\varepsilon_{c}$ \\
\hline Skin & $7.98-j 10.91$ \\
Fat & $2.51-j 0.84$ \\
Muscle & $12.85-j 15.74$ \\
Pure water $\left(20^{\circ} \mathrm{C}\right)$ & $11.9-j 19.5$ \\
\hline
\end{tabular}


$\varepsilon_{c}$ : Complex permittivity;

$\varepsilon_{r}:$ Relative permittivity;

$\omega$ : Angular velocity measured in radians per second;

$\varepsilon_{o}:$ Free space permittivity, which equals $8.85 \times 10^{-12} \mathrm{~F} / \mathrm{m}$.

There are two models to simulate the human body shadowing in an indoor environment. The first model is known as "Phantom Human" and the other is called "Salty-Lite". In general, the human body is represented as a parallelepiped circumscribed with Salty-Cylinder model. The lengths of sides of the basic rectangle will be equal to $0.305 \mathrm{~m}$, the height is equal to $1.7 \mathrm{~m}$, and the thickness is equal to $0.305 \mathrm{~m}$ $[5,23]$. In this work, the human body represented as a parallelepiped circumscribed which contains skin, fat, muscles, and a cylinder with a pure water at $20^{\circ} \mathrm{C}$, as shown in Fig. 13.

\section{Simulation Results Using "Wireless InSite" Software}

"Wireless InSite" is the software tool used to give the closest scenario for signals propagating from $\mathrm{Tx}$ (transmitter) to $\mathrm{Rx}$ (Receiver) inside the area of study [24]. The $S_{11}$ and $S_{21}$ parameters values for designed SL FSS have been exported to "Wireless InSite".

A small area of interest with only one room which has been investigated with and without SL FSS to check its effect on reflecting the frequency of interest of this study and improving the received signal power in presence of humans. The comparison between scenarios, with and without SL FSS, has shown how the SL FSS affected the signal propagation and enhanced the received signal power.

\section{Open Area Scenario}

One room with dimensions $15 \times 10 \mathrm{~m}$ is considered. SL FSS is attached on the wall scenario in order to band-stop $60 \mathrm{GHz}$. SL FSS is placed away from the wall by $\lambda / 10$ in order to eliminate the coupling effect [11].

The area of interest has an omnidirectional antenna as a route of Rx's with a sensitivity of $-64 \mathrm{dBm}$ [25]. The height of Rx's is $1 \mathrm{~m}$ and the spaces between the grid antennas of Rx's are $0.5 \mathrm{~m}$. Tx has directional antenna with input power $10 \mathrm{dBm}$ and gain of $14 \mathrm{dBi}$. The height of Tx is $2.5 \mathrm{~m}$ above the floor which is mounted on the ceiling. The electric parameters of the materials used in the room scenario are presented in Table 2 [24].

\section{A. First Case without SL FSS Wallpaper}

Fig. 14 shows the 2D view of the open area scenario with many persons standing randomly around. As well, this scenario consists of Tx and five route Rx's, each one has six points, the whole distance between them is $0.5 \mathrm{~m}, 0.1 \mathrm{~m}$ between each pair, that were arranged behind the person.

As shown in Fig. 15, the black parallelepiped represents a human which stands in the room with no SL FSS attached on the wall (normal case).

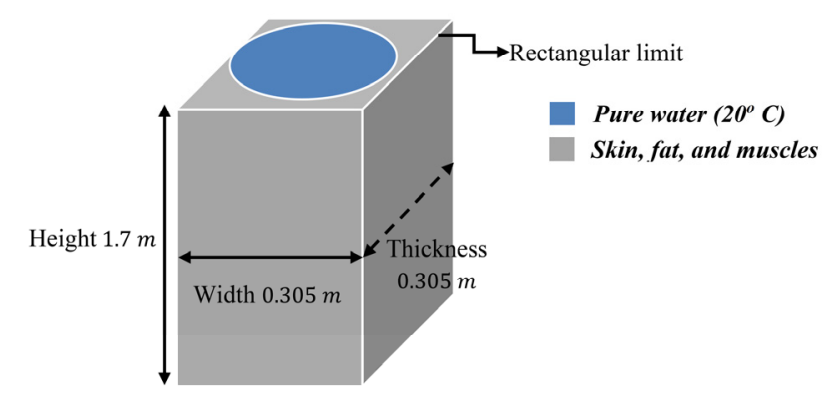

Fig. 13 The human model used in this study.

Table 2 Electrical parameters used for building interfaces.

\begin{tabular}{lllll}
\hline The user interface & Material & Relative electrical permittivity, $\varepsilon_{\mathrm{r}}$ & Conductivity, $\sigma(\mathrm{S} / \mathrm{m})$ & Thickness, $(\mathrm{m})$ \\
\hline Walls & Brick & 4.44 & 0.001 & 0.150 \\
Doors & Wood & 5 & 0 & 0.03 \\
Windows & Glass & 2.4 & 0 & 0.003 \\
Ceiling \& floor & Concrete & 15 & 0.015 & 0.3 \\
\hline
\end{tabular}




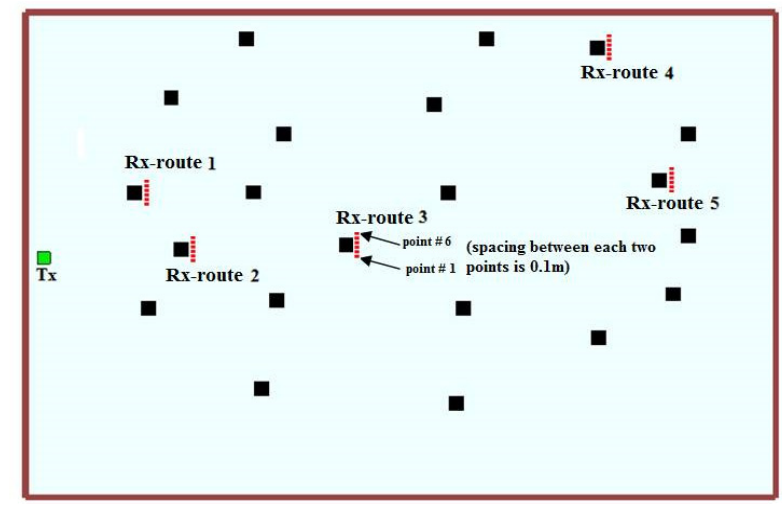

Fig. 14 2D view for the open area scenario.

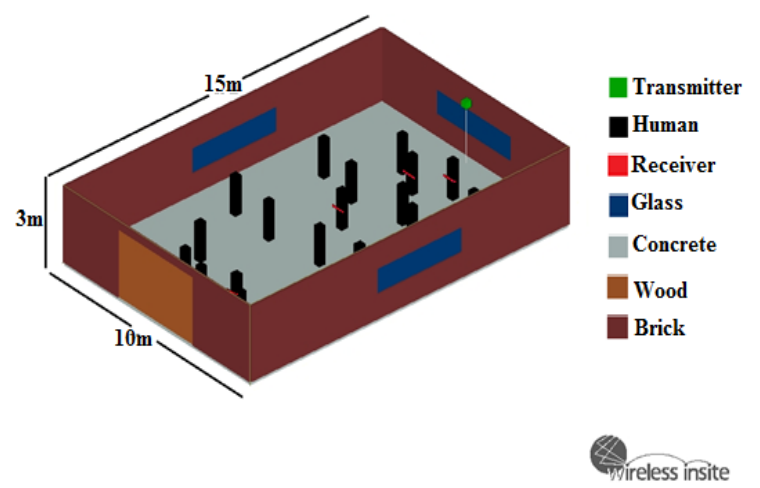

Fig. 15 3D view for the open area scenario with no SL FSS.

By tracking the strength of the propagating signal from Tx to Rx's, as an example Rx-route 3, the received $60 \mathrm{GHz}$ signal power is very low because of the effect of human which will attenuate the propagating signal, as shown in Fig. 16.

\section{B. Second Case with SL FSS Wallpaper}

In this case, SL FSS is attached on the wall, as shown in Fig. 17. The SL FSS here will work as a good reflector for the $60 \mathrm{GHz}$ signal, and denies the signal getting out of the room, which will minimize the effect of human in attenuating $60 \mathrm{GHz}$ signal. By tracking the propagating signal from $\mathrm{Tx}$ to $\mathrm{Rx}$-route 3 , the received $60 \mathrm{GHz}$ signal power will be much higher than first case where no SL FSS is around, as shown in Fig. 18.

To prove the enhancement in the received signal power by adding SL FSS, comparisons between presence and absence of the SL FSS at every Rx point have been illustrated in Figs. 19-23. As shown in
Fig. 19, the received power for Rx-route 1, without SL FSS, is varied from -77.9 to $-57.1 \mathrm{dBm}$, while with SL FSS case is varied from -74.7 to $-50.4 \mathrm{dBm}$, where all points have been enhanced.

As shown in Fig. 20, the received power with the no presence of SL FSS is varied from -67.4 to -63.3 $\mathrm{dBm}$, while in the presence of SL FSS is varied from -59.8 to $-52.1 \mathrm{dBm}$.

As shown in Fig. 21, the received power without SL FSS case is varied from -63.5 to $-63 \mathrm{dBm}$, while in case of SL FSS around is varied from -63.8 to -57.3 $\mathrm{dBm}$.

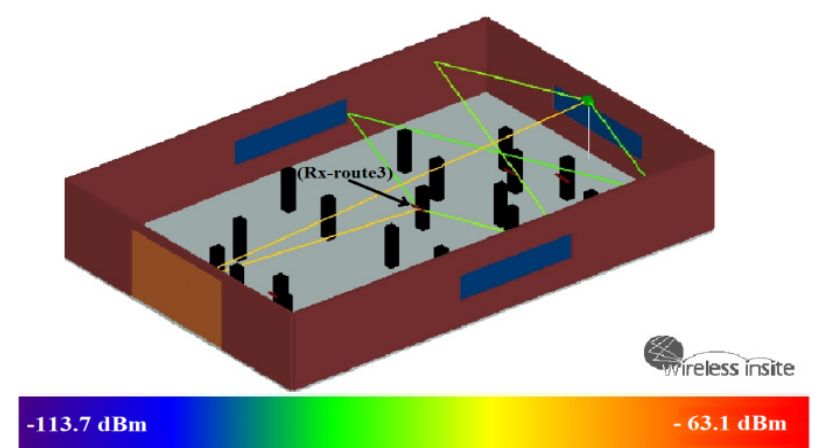

Fig. 16 The power of maximum 3 propagating paths from Tx to Rx-route 3 without SL FSS.
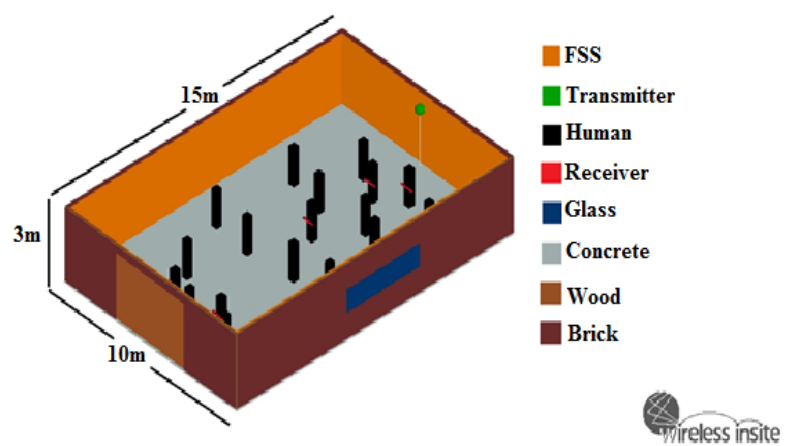

Fig. 17 3D view for the open area scenario with SL FSS.

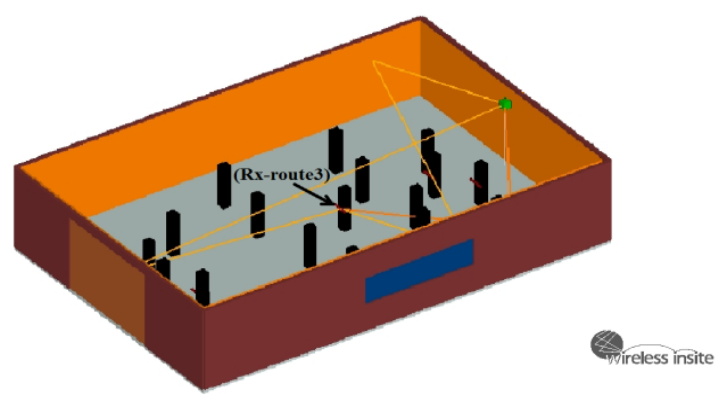

$-114.8 \mathrm{dBm}$ $-56.6 \mathrm{dBm}$

Fig. 18 The power of maximum 3 propagating paths from Tx to Rx-route 3 with SL FSS. 


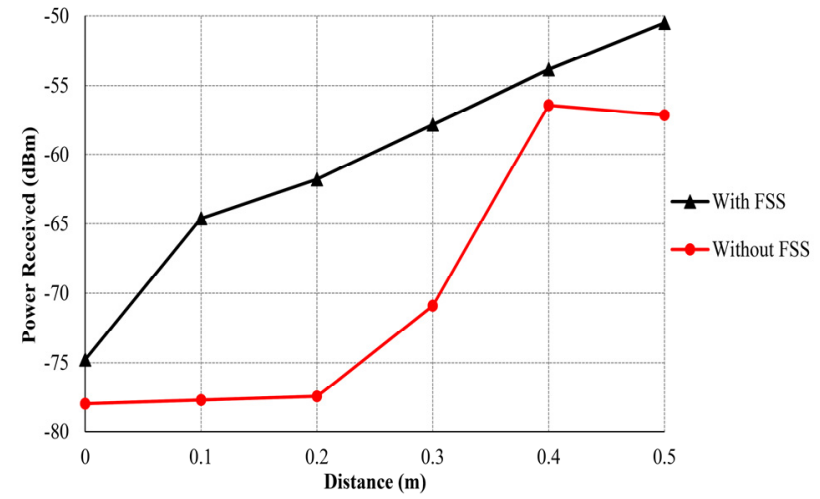

Fig. 19 Received power for Rx-route 1.

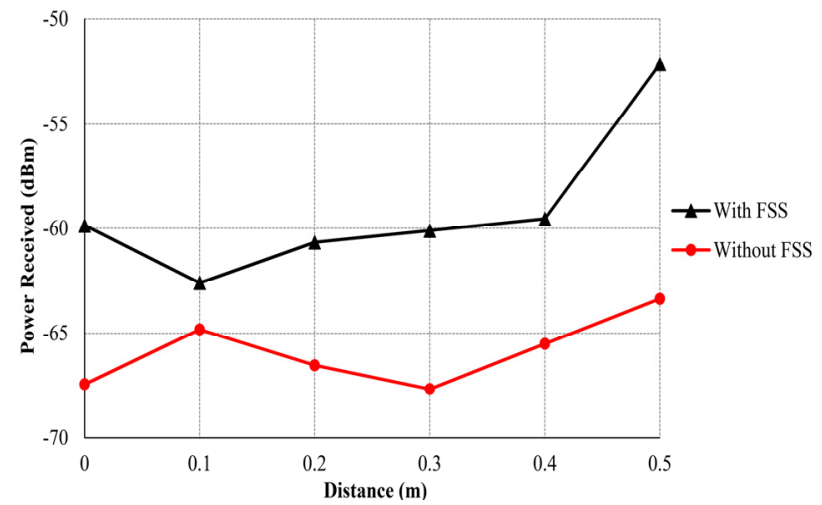

Fig. 20 Received power for Rx-route 2.

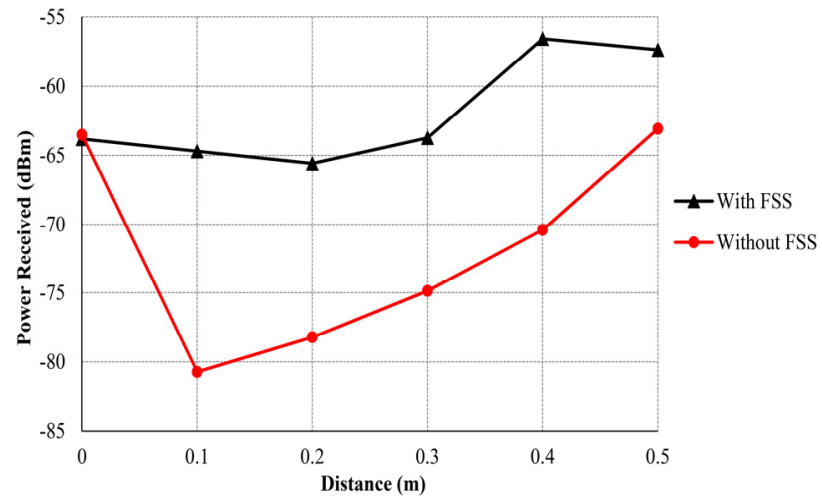

Fig. 21 Received power for Rx-route 3.

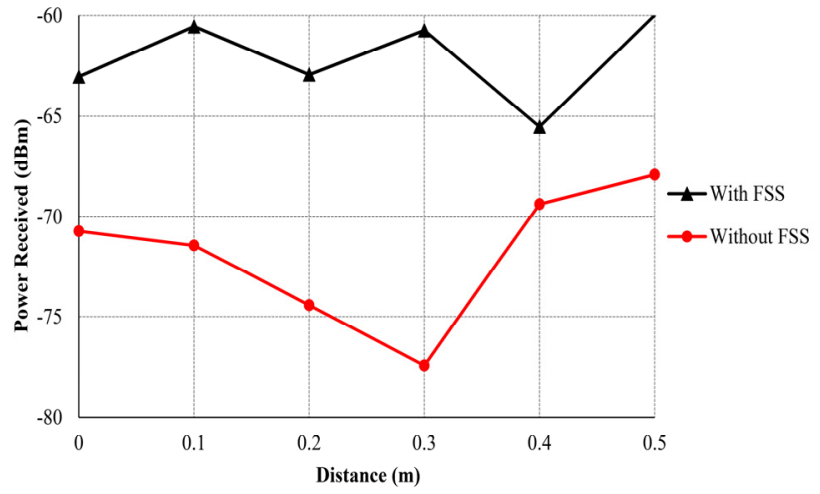

Fig. 22 Received power for Rx-route 4.

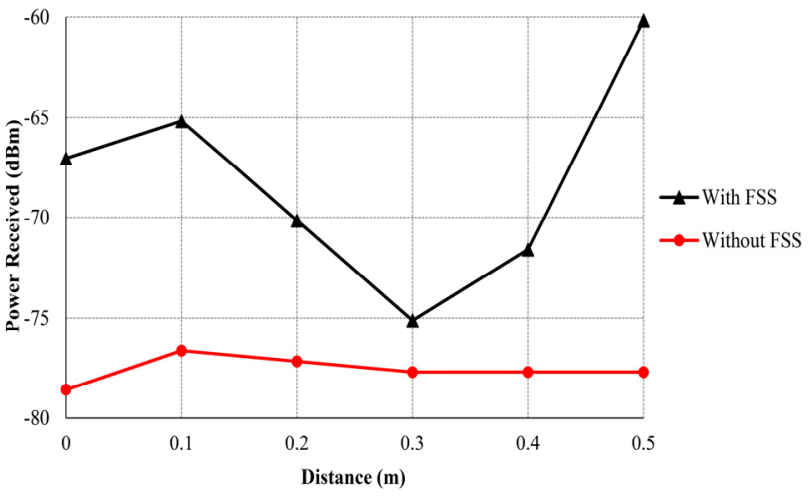

Fig. 23 Received power for Rx-route 5.

Even for far Rx routes like Rx-route 4 and Rx-route 5, as shown in Figs. 22 and 23 respectively, the power of the received signal in the presence of SL FSS has been enhanced. Where the received power without SL FSS for Rx-rout 4, as shown in Fig. 22, is varied from -70.7 to $-67.9 \mathrm{dBm}$, while with SL FSS is varied from -63 to $-59.9 \mathrm{dBm}$. For Rx-route 5 without SL FSS, the received power is varied from -78.5 to $-77.6 \mathrm{dBm}$, while with SL FSS case is varied from -67 to -60.1 dBm, as shown in Fig. 23.

\section{Delay Spread}

Mean time of arrival and delay spread are the statistical measures of the time dispersion of the channel [26]. Both of them are explained mathematically in details in the following equations $[24,27]$. The time of arrival is:

$$
t_{i}=\frac{L_{i}}{C}
$$

where:

$t_{i}$ : Time of arrival;

$L_{i}$ : Total geometrical path length;

$\mathrm{C}$ : The speed of light in free space.

and the mean time of arrival is:

$$
\overline{\mathrm{t}}=\frac{\sum_{\mathrm{i}=1}^{N} \mathrm{p}_{\mathrm{i}} \mathrm{t}_{\mathrm{i}}}{\mathrm{p}_{\mathrm{R}}}
$$

and the RMS (root mean square) delay spread is:

$$
\sigma=\sqrt{\frac{\sum_{\mathrm{i}=1}^{N_{p}} p_{i} t_{i}^{2}}{P_{R}}-\bar{t}^{2}}
$$

where:

$P_{R}$ : The averaged received power; 
$N_{p}$ : The number of paths;

$P_{i}$ : The time averaged power in watts of the $\mathrm{i}^{\text {th }}$ path.

In this paper, the open area scenario is studied in the presence and absence of SL FSS, in order to show the effect of adding SL FSS to the mean time of arrival and delay spread values. In order to accomplish this, two $\mathrm{Rx}$ routes have been taken which are Rx-route 1 and Rx-route 3.

As shown in Fig. 24, the average of the mean time of arrival in FSS case for Rx-route 1 was $37.5 \mathrm{~ns}$. The minimum value was $13.2 \mathrm{~ns}$, and the maximum value was $61.8 \mathrm{~ns}$. While without FSS, the average was $25.85 \mathrm{~ns}$. The minimum value was $10.7 \mathrm{~ns}$ and the maximum value was $41 \mathrm{~ns}$. As a result, these values are within the acceptable range for $60 \mathrm{GHz}$ [25]. Also, the mean time of arrival with FSS is higher than no FSS due to the richness in multipath in FSS case.

As shown in Fig. 25, the average of mean time of arrival in FSS case for Rx-route 3 was 53.15 ns, while with no FSS case was 51.1 ns.

As shown in Fig. 26, the average of delay spread for Rx-route 1 in FSS case was 20.9 ns, while with no FSS was 13.2 ns.

As shown in Fig. 27, the average of delay spread in FSS case for Rx-route 3 was 15.5 ns, while with no FSS was 10.8 ns.

\section{Corridor Scenario}

In this scenario, an L-shape corridor will be studied with a directional horn antenna at $\mathrm{Tx}$ and $\mathrm{Rx}$. The input power for $\mathrm{Tx}$ is $10 \mathrm{dBm}, \mathrm{Tx}$ height is $1.5 \mathrm{~m}, \mathrm{Rx}$ height is $2 \mathrm{~m}, \mathrm{Rx}$ is $0.5 \mathrm{~m}$ route, and the spacing between each point is $0.1 \mathrm{~m}$. The total number of points is six. Simulations have been done using "Wireless InSite" and performed without SL FSS, with SL FSS and PEC, and with $45^{\circ}$ inclined PEC plane, as shown in Fig. 28. The dimension of SL FSS and PEC is $3 \times 3 \mathrm{~m}$. These cases were studied in order to show the impact of adding SL FSS and PEC on the received signal power.
The first study case is a corridor without SL FSS, as shown in Fig. 29. The second study case is a corridor with SL FSS and PEC, as shown in Figs. 30 and 31 respectively.

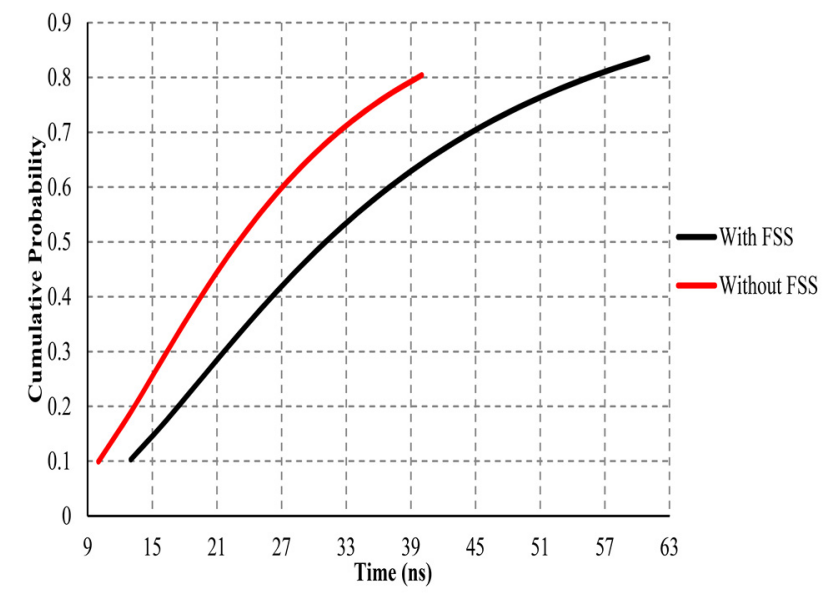

Fig. 24 Mean time of arrival for Rx-route 1.

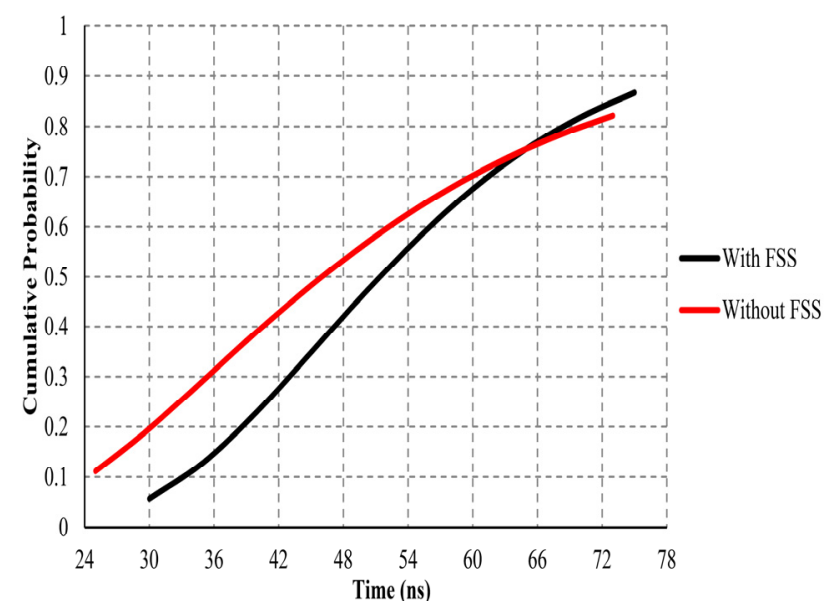

Fig. 25 Mean time of arrival for Rx-route 3.

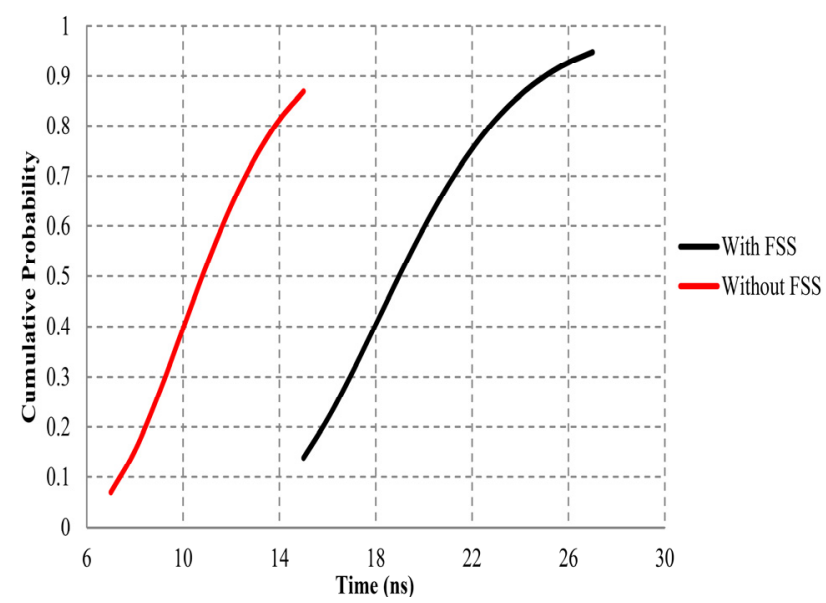

Fig. 26 RMS delay spread for Rx-route 1. 


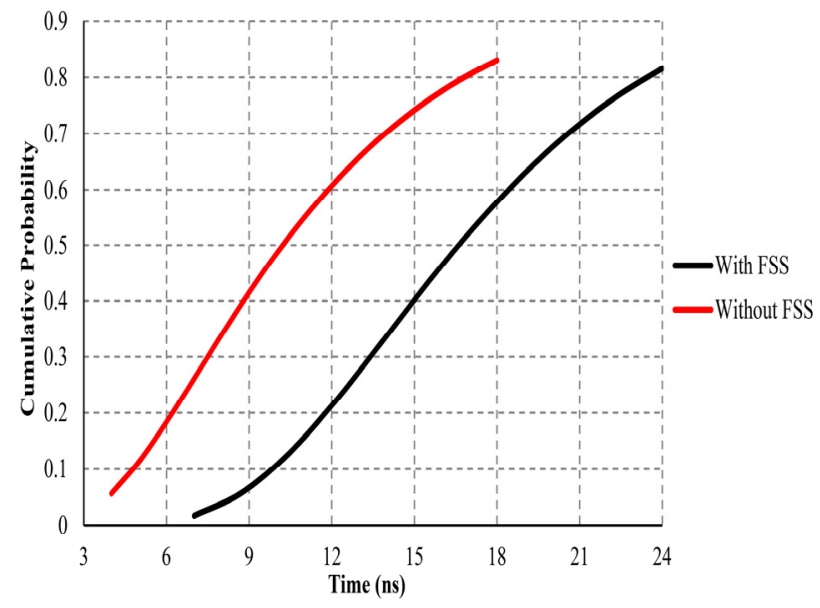

Fig. 27 RMS delay spread for Rx-route 3.

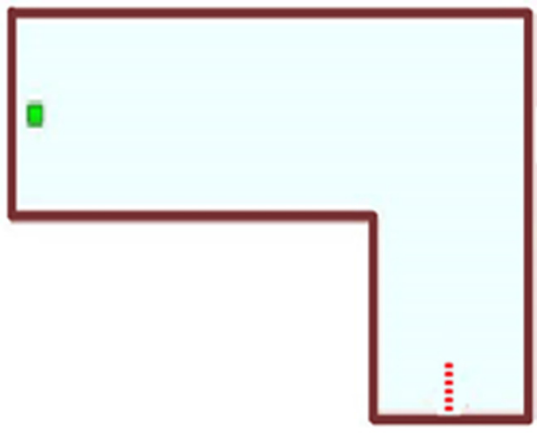

Transmitter

Receiver

(a) Without SL FSS.

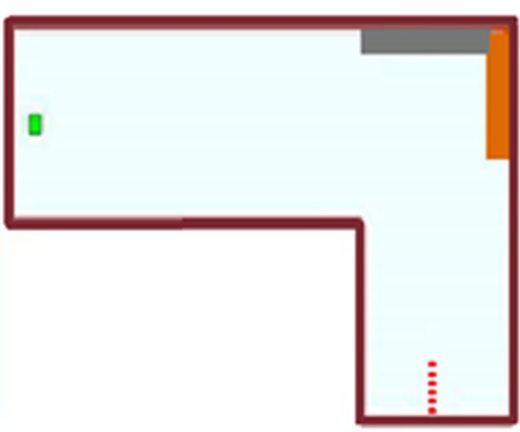

FSS

Transmitter

Receiver

PEC

(b) With SL FSS and PEC.

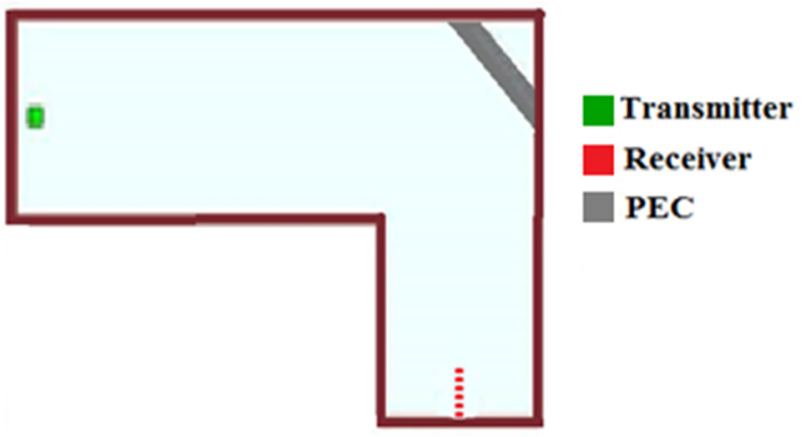

(c) With $45^{\circ}$ inclined PEC plane.

Fig. 28 2D view of $L$-shape corridor scenario.

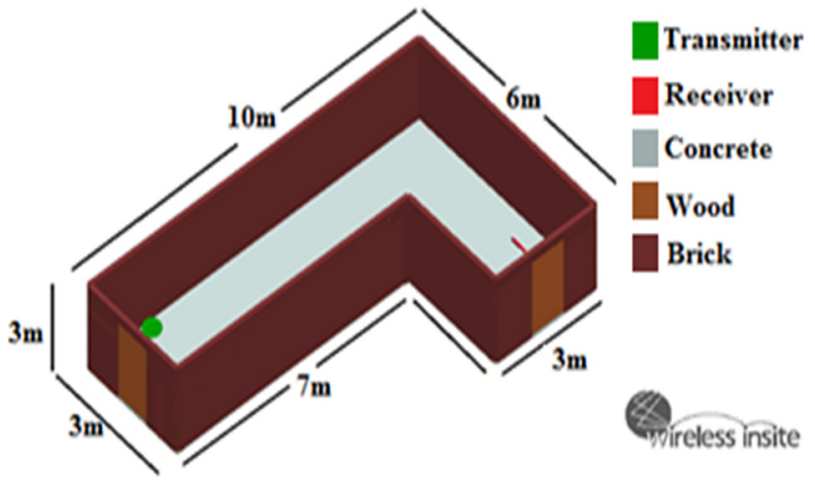

Fig. 29 3D view of $L$-shape corridor scenario without SL FSS.

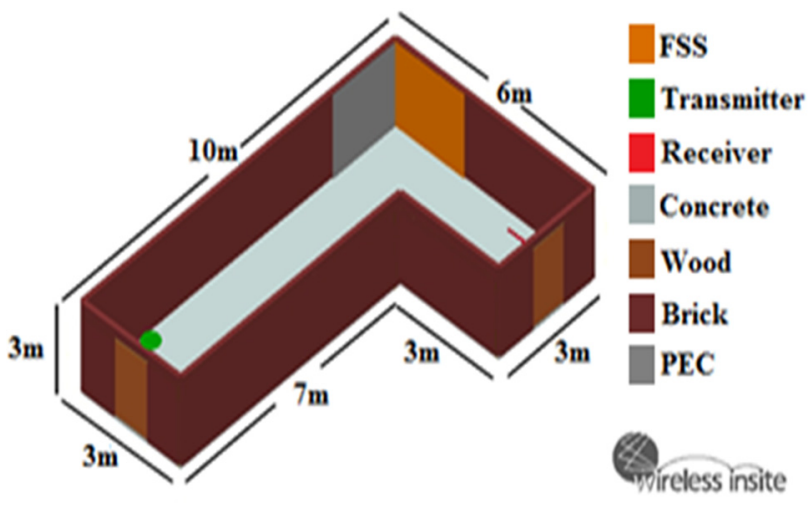

Fig. 30 3D view of $L$-shape corridor scenario with SL FSS and PEC.

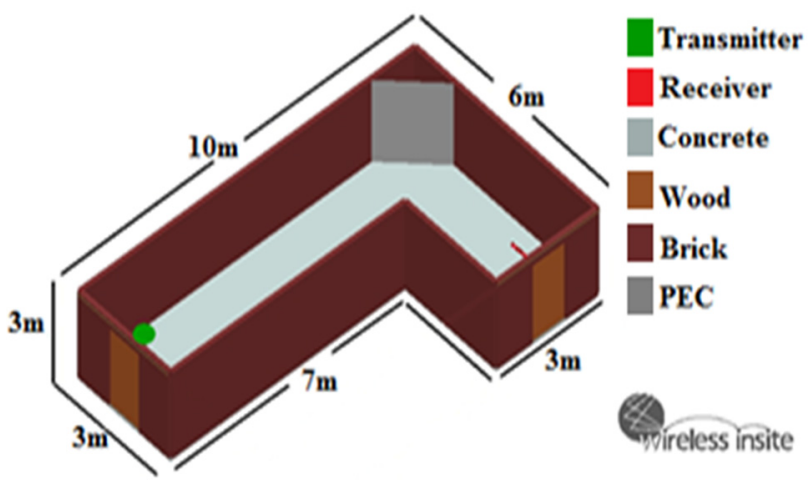

Fig. 31 3D view of $L$-shape corridor scenario with $45^{\circ}$ inclined PEC.

As shown in Fig. 32, the power of the received signal without SL FSS case is varied from -80.9 to $-65.9 \mathrm{dBm}$, while with SL FSS and PEC case is varied from -59.9 to $-51.1 \mathrm{dBm}$. In the case of inclined PEC, the power of the received signal is varied from -57.2 to $-49.9 \mathrm{dBm}$. The average of the received signal power without SL FSS, with SL FSS and PEC, and with inclined PEC case was $-68.542,-57.295$, and $-51.609 \mathrm{dBm}$ respectively. 


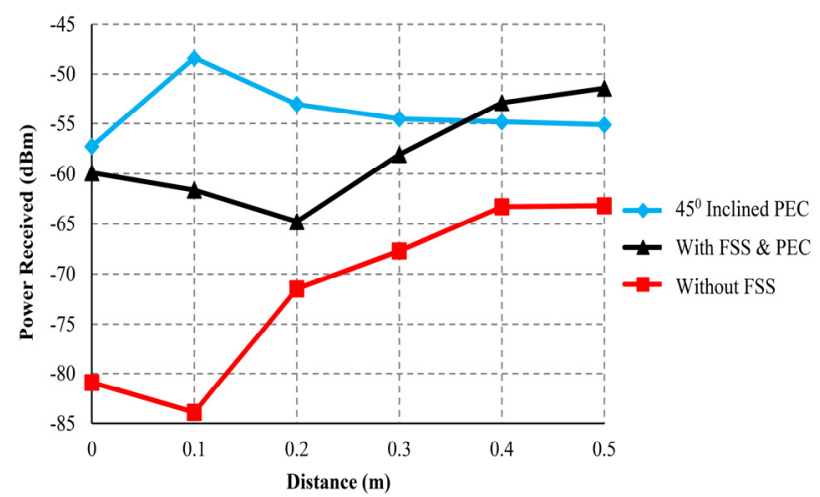

Fig. 32 Received power for L-shape corridor scenario.

Depending on power averages showed before, the $45^{\circ}$ inclined PEC plane case was the best, but due to the structural considerations, the inclined PEC reduces the area of the corridor. So, SL FSS with PEC case is more suitable and applicable choice.

\section{Conclusion}

Enhancing the received signal power for $60 \mathrm{GHz}$ indoor wireless propagation environment in order to minimize the effect of human in blocking $60 \mathrm{GHz}$ signal was investigated and demonstrated in this paper.

The frequency response of SL FSS wall developed in this paper showed a good capability to deal with TE-mode and TM-mode waves.

SL FSS was used as a band-stop filter to block 60 $\mathrm{GHz}$ propagation from outside/inside the area of interest and as a perfect reflector from inside. One room was investigated to simulate the SL FSS action and how it affects the signal propagation. The received signal power has been enhanced. Because of, it provided multipath propagation due to the variety of signal paths where diminished the influence of human shadowing. With the direct path obstructed by a human body, the signal power enhancement ranged from 3 to $15 \mathrm{~dB}$, depending on the position of the human body and the angle of arrival of the path.

Comparing simulations for a normal and a modified wall, as the stronger reflected paths from the modified wall have time delays more than to those of the dominant paths from the normal wall. For the same reason, in the presence of the modified wall, an increased portion of the total received power arrives at times similar to the mean delay time. Therefore, the RMS delay spread is increased slightly, which is still within an acceptable range.

Also, a corridor scenario has been studied to prove the contribution of this paper, three cases have been stated; without SL FSS, with SL FSS and PEC, and with $45^{\circ}$ inclined PEC plane. Results indicated that the $45^{\circ}$ inclined PEC plane case was the best, but due to the structural considerations, the SL FSS with PEC case is more suitable and applicable choice.

\section{References}

[1] Choi, S. W., Kim, I. G., and Jae, M. A.2013. "Architecture of Baseband Modem Board for IEEE 802. 11ad Technology." Presented at the 2013 International Conference on ICT Convergence (ICTC).

[2] Guo, N., Robert, C., Qiu, S. M., Mo, S., and Kazuaki, T. 2007. "60-GHz Millimeter-Wave Radio: Principle, Technology, and New Results." EURASIP Journal on Wireless Communications and Networking 2007 (1):48-8.

[3] Smulders, P. F. M. 2003. "60 GHz Radio: Prospects and Future Directions." In Proceedings Symposium IEEE Benelux Chapter on Communications and Vehicular Technology.

[4] Singh, S. 2009. "Medium Access Control and Bandwidth Allocation in Millimeter-Wave and WiFi Networks." PhD diss., UNIVERSITY OF CALIFORNIA Santa Barbara.

[5] Qasem, N. 2014. "Enhancing Wireless Communication System Performance through Modified Indoor Environments." PhD diss., (C) Nidal Qasem.

[6] Kartal, M., Bora, D., and Ismail, G. 2011. "Design for the Structural Surface Material Enabling Shielding for Interference Mitigation within the Buildings in the Unlicensed 2.4 GHz ISM Band." Presented at the 2011 $\mathrm{XXX}^{\text {th }}$ URSI General Assembly and Scientific Symposium.

[7] Taylor, P. S., Edward, P., and John, C. B. 2011. "An Active Annular Ring Frequency Selective Surface.” IEEE Transactions on Antennas and Propagation 59(9):3265-71.

[8] AlKayyali, H., and Nidal Q. 2013. "Convoluted Frequency Selective Surface Wallpaper to Block the Industrial, Scientific, and Medical Radio Bands Inside Buildings." American Academic \& Scholarly Research 


\section{Overcoming the Influence of Human Shadowing and Obstacles via Modified Building Using Frequency Selective Wallpapers for $60 \mathrm{GHz}$}

Journal 5 (3): 106.

[9] Qasem, N., and Rob, S. 2010. "Studies on enhancing wireless signal for indoor propagation." Presented at the IEEE Antennas and Propagation Conference (LAPC), Loughborough,2010.

[10] Qasem, N., and Rob, S. 2009. "Frequency Selective Wall for Enhancing Wireless Signal in Indoor Environments." Presented at the IEEE Antennas \& Propagation Conference, 2009. LAPC 2009. Loughborough.

[11] Dawod, D., and Nidal, Q. 2015. "Enhancing the Capacity of MIMO Systems via Modified Building Using Frequency Selective Wallpapers.” Presented at the 2015 6th International Conference on Information and Communication Systems (ICICS).

[12] Qasem, N., and Rob, S. 2011. "Indoor Band Pass Frequency Selective Wall Paper Equivalent Circuit \& Ways to Enhance Wireless Signal." Presented at the Antennas and Propagation Conference (LAPC), Loughborough.

[13] Mias, C., Tsakonas, C., and Oswald, C.2001. "An Investigation into the Feasibility of Designing Frequency Selective Windows Employing Periodic Structures." Radiocommunications Agency Final Rep.

[14] Anderson, I. 1975. "On the Theory of Self-resonant Grids." Bell System Technical Journal 54(10):1725-31.

[15] Lee, C. K., and Langley, R. J. 1985. "Equivalent-Circuit Models for Frequency-Selective Surfaces at Oblique Angles of Incidence." In IEEE Proceedings $H$ (Microwaves, Antennas and Propagation),395-99, IET Digital Library.

[16] Munk, B. 2005. A Frequency Selective Surfaces: Theory and Design. John Wiley \& Sons.

[17] Hamdy, S. M. A., and Edward A. P. 1982. "Current Distribution on the Elements of a Square Loop Frequency Selective Surface." Electronics Letters 18(14): 624-26.

[18] Marcuvitz, N. 1951.Waveguide handbook (No. 21). Iet.

[19] Savia, S. B., and Parker, E. A.2003. "Equivalent Circuit
Model for Superdense Linear Dipole FSS.” In IEEE Proceedings of Microwaves, Antennas and Propagation, 37-42, IET.

[20] Anyonopoulos, C., and Edward A. P. 1998. "Design Procedure for FSS with Wide Transmission Band and Rapid Rolloff." In IEEE Proceedings of Microwaves, Antennas and Propagation, 508-10, IET.

[21] Chahat, N., Maxim, Z., Ronan, S., and Stanislav, A.2012. "New Method for Determining Dielectric Properties of Skin and Phantoms at Millimeter Waves Based on Heating Kinetics." IEEE Transactions on Microwave Theory and Techniques 60(3):827-32.

[22] Gustafson, C., and Fredrik, T. 2012. "Characterization of $60 \mathrm{GHz}$ Shadowing by Human Bodies and Simple Phantoms." Presented at the 2012 6th European Conference on Antennas and Propagation (EUCAP).

[23] Qasem, N., and Rob, S.2012. "Overcoming the Influence of People Shadowing and Enhancing MIMO Capacity Systems via Modified Environments." Presented at the Antennas and Propagation Conference (LAPC), 2012 Loughborough.

[24] Remcom. (2016, April 15). Retrieved from http://www.remcom.com/electromagnetic-applications/.

[25] Zhu, Y. B., Zhang, Z. B., Marzi, Z., Chris, N., Upamanyu, M., Ben, Y. Z., and Haitao, Z. 2014. "Demystifying 60ghz Outdoor Picocells." In Proceedings of the 20th Annual International Conference on Mobile Computing and Networking,5-16.

[26] Xu, H., Vikas, K., and Theodore, S. R. 2002. "Spatial and Temporal Characteristics of $60-\mathrm{GHz}$ Indoor Channels." IEEE Journal on Selected Areas in Communications20(3):620-30.

[27] Qasem, N, and Rob, S. 2012. "Parametric Studies in Enhancing Indoor Wireless Communication System via Environmental Modification." Presented at the Antennas and Propagation Conference (LAPC), Loughborough. 